\title{
Interoperabilidade entre acervos digitais de arquivos, bibliotecas e museus: potencialidades das tecnologias de dados abertos interligados ${ }^{1}$
}

Carlos Henrique Marcondes

Professor, mestre e doutor em Ciência da Informação, pesquisador do CNPq

http://dx.doi.org/10.1590/1981-5344/2735

A Web Semântica e os dados abertos interligados propiciaram a publicação de acervos digitais de arquivos, bibliotecas e museus diretamente na Web sem a intermediação de sistemas gerenciadores de catálogos e colocou a questão da integração destes acervos, sua interoperabilidade. Neste contexto ampliam-se as demandas pela preservação da semântica dos conteúdos disponibilizados, garantida anteriormente pelos sistemas de catálogos. Ao mesmo tempo estas tecnologias viabilizam novos tipos de relações culturamente significativas que podem ser estabelecidas entre objetos digitais pertencentes a estes acervos. Que desenvolvimentos tecnológicos e metodológicos são necessários para tirar partido destas tecnologias? Este trabalho tem como objetivo recolocar a questão da interoperabilidade entre acervos digitais a luz das possibilidades trazidas pelas tecnologias da Web Semântica e dos dados abertos interligados. Como metodologia foi utilizada a revisão do estado da arte aspectos teóricos, técnicos, projetos, experiências - a repeito da questão da interoperabilidade e utilização de parâmetros de análise homogeneidade/heterogeneidade dos acervos a serem integrados e mecanismos para garantir que os significados originais dos conteúdos nos sistemas isolados seriam preservados. Como resultados o papel dos modelos conceituais e a investigação das possíveis relações semânticas entre estes acervos são destacados para alcançar a interoperabilidade, bem como o papel, neste contexto, da curadoria digital.

\footnotetext{
${ }^{1}$ Esta é uma versão revista e ampliada de trabalho apresentado no XV EDICIC, Madrid, Espanha, 2015.
} 
Palavras-chave: Acervos digitais; Arquivos; Bibliotecas; Museus; Interoperabilidade; Relações semânticas; Integração; Dados abertos interligados; Curadoria digital.

\section{Interoperability between digital collections in archives, libraries and museums: potentialities of linked open data technologies}

The Semantic Web and linked open data enable the publication of digital collection of archives, libraries and museum in the Web eliminating the intermediation of catalog management systems and poses the question of their integration, namely interoperability. In such context the requirements for preserving the original record's semantics, which are guaranteed by catalog systems, are reinforced. At the same time these technologies enable new cultural relevant relations to be settled between digital objects of different cultural heritage collections. What technological and methodological developments are needed to exploit the facilities brought these technologies? This paper put back the issues related to interoperability between such digital collections in light of the facilities offered by these technologies. It uses as methodology a state of the art review of theoretical and technical issues, projects, cases, etc., related to interoperability and analyses interoperability solutions based on two parameters, homogeneity/heterogeneity and the preservation of original semantic of the collections to be made interoperable. As results the role of semantic models and research on possible semantic relationships between such collections are highlighted as means to achieve interoperability, as well as, in such a context, of digital curation.

Keywords: Digital collections; Archives; Libraries; Museums; Interoperability; Integration; Linked open data; Digital curation.

Recebido em 02.01.2016 Aceito em 01.06.2016 


\section{Introdução}

Instituições como arquivos, bibliotecas e museus têm tido, por séculos, a missão de manterem, preservarem e disseminarem os acervos de memória e cultura das sociedades onde estão inseridas. Embora tenham a preservação da memória e da cultura como missão comum, estas instituições têm também muitas características e procedimentos específicos. Diferenças de gestão e curadoria de seus acervos, diferenças na conceituação dos objetos dos acervos a descrever (diferentes "objetos" a representar: livros, fundos e séries, objetos museológicos únicos), idiossincrasias relacionadas a tradições profissionais distintas, padrões diferentes, etc., tornam a integração dos acervos dessas instituições um desafio conceitual e tecnológico.

A emergência das tecnologias de informação, bem como uma demanda característica das modernas sociedades democráticas do fim do século $X X$, de que instituições públicas prestem contas e justifiquem os investimentos da sociedade em mantê-las, fez com que as instituições de memória se voltassem cada vez mais para disseminação de seus acervos, ao invés de somente preservá-los. Esta mudança é conhecida como "paradigma pós-custodial" (RIBEIRO, 2011), as instituições de memória e cultural passam a não ter somente a missão de custodiar, preservar e manter seus acervos, mas também disseminá-los para que se constituam em recursos de memória, de história, de cultura, de educação e de cidadania das sociedades onde estão inseridas.

As tecnologias da informação sempre foram usadas para agregar diferentes acervos, potencializando suas sinergias e complementaridades e provendo melhores serviços aos seus usuários. Bibliotecas foram pioneiras neste sentido, motivadas pela necessidade de compartilhar o trabalho de catalogação. Tecnologias e padrões específicos foram desenvolvidos com esta finalidade. $O$ formato Machine-Readable Cataloging (MARC) ${ }^{2}$ - data de fins da década de 60 do século XX (ROBREDO; CUNHA, 1986). O protocolo Z39.50 é de fins da década de 80. O MARC é um formato de intercâmbio de dados bibliográficos/catalográficos que permite formar uma base de dados ou catálogo coletivo comum. O Z39.50 é um protocolo que permite a usuários consultarem diferentes catálogos, hospedados em servidores diferentes e mantidos por instituições diferentes, como se fossem um único catálogo "virtual".

Mais recentemente o protocolo Open Archives Initiative Protocol for Metadata Harvesting (OAI-PMH) ${ }^{3}$, juntamente com 0 padrão de metadados Dublin Core ${ }^{4}$, oferecem uma solução simples para a coleta automática de metadados e sua agregação numa base de dados comum que permita consulta unificada a diferentes acervos. Embora tenham

\footnotetext{
${ }^{2}$ MARC. Disponível em: <http://www.loc.gov/marc/>. Acesso em : 18 jun. 2016.

${ }^{3}$ OAI-PMH. Disponível em: <https://www.openarchives.org/pmh/>. Acesso em : 18 jun. 2016.

${ }^{4}$ Dublin Core. Disponível em: <http://dublincore.org >. Acesso em : 18 jun. 2016.
} 
havido tentativas, em especial nos EUA e Canadá, de usar o MARC para o tratamento de acervos arquivísticos (WALCH, 1994), a simplicidade e generalidade do padrão de metadados Dublin Core, para o qual podem ser mapeados registros bibliográficos, arquivísticos e museológico, quando associado ao protocolo OAI-PMH, colocou na ordem do dia a questão da integração entre acervos arquivísticos, bibliográficos e museológicos. Estas tecnologias e padrões têm sido conhecidos na literatura sob a denominação de "interoperabilidade".

As soluções tecnológicas de interoperabilidade, desde o formato MARC da década de 60 do século $X X$, sempre estiveram baseadas na troca de metadados, na sua agregação em uma base de dados comum e na operação desta base por programas gerenciadores de catálogos. Nos esforços para se alcançar a interoperabilidade entre sistemas distintos, busca-se, cada vez com mais ênfase, a troca de dados de forma significativa (NILSSON; BAKER; JOHNSTON, 2009). Estes autores enfatizam diferentes estágios de interoperabilidade com o objetivo de se garantir que seja preservada a semântica original dos conteúdos trocados.

No entanto o cenário de interoperabilidade baseado na troca de metadados, predominante deste a $2^{a}$. metade do século $X X$, parece estar mudando a partir do surgimento das tecnologias da Web Semântica e dos dados abertos interligados. Estas tecnologias tornam viável hoje a integração de acervos digitais publicados na Web de arquivos, bibliotecas e museus, de novas e inusitadas maneiras. Em especial, estas tecnologias, em especial a representação utilizando triplas - Resorce Description Framework (RDF, 2014) -, ao contrário das soluções anteriores de interoperabilidade, baseadas na troca de metadados, sua agregação em bases de dados comuns e sua operação por programas gerenciadores de catálogos, tornam possível agora que acervos digitais sejam publicados diretamente na Web.

O modelo de triplas RDF pressupõe sempre uma afirmação com um sujeito - o recurso que esta sendo descrito, um predicado referente a este recurso e o objeto deste predicado, assim: "A pagina web www.joaodasilva.org (recurso de informação digital) tem como autor (predicado) João da Silva (objeto)". As triplas RDF e seus elementos têm identificadores/endereços persistentes - URI -, podendo assim tanto fazer links para, ou terem links desde quaisquer outros recursos também publicados na Web. Cada um dos componentes de uma tripla RDF pode também ter seu significado qualificado e tornado mais preciso, para além de ser meramente sujeito, predicado ou objeto de uma afirmação, ao fazer referência a um vocabulário específico. Assim, poderia ser acrescentado à afirmação de que "A pagina web www.joaodasilva.org (recurso) tem como autor (predicado) João da Silva (objeto)", a referência ao elemento do vocabulário de metadados Dublin Core, dc:creator (predicado) João da Silva. Ao ser publicada com esta referência um documento RDF ficaria com seu significado (o significado de cada elemento de suas triplas) mais preciso, a menção a seu criador teria o significado preciso tomado do elemento Dublin Core creator. 
Além disso, na tecnologia dos catálogos disponibilizados na Web, comuns nas páginas de instituições arquivísticas, bibliotecas e museus pelo mundo afora, os programas gerenciadores destes catálogos tinham papel preponderante de proporcionar semântica a estes conteúdos; eram eles que faziam com que determinada cadeia de caracteres fosse mostrada aos usuários como o Autor ou o Título de uma publicação. Os programas gerenciadores de catálogos faziam isto às custas de limitarem o acesso a estes conteúdos através da (necessária) intermediação que estes programas fazem. Ao serem publicados diretamente na Web através das tecnologias de dados abertos interligados estes conteúdos tornam-se independentes dos programas gerenciadores de catálogos. Uma visão bastante completa de todas as questões envolvidas na publicação de dados abertos interligados por ser encontrada em Heath e Bizer (2011)

Com estas tecnologias a semântica dos conteúdos publicados tem que ser agregada por dispositivos conhecidos como modelos conceituais, ontologias e/ou vocabulários semânticos (RODRÍGUEZ; GREENBERG, 2012), que podem ser representados em formato legível por programas e associados às triplas RDF, de modo a especificar univocamente 0 significado dos predicados e objetos de cada tripla. A medida que "links" são feitos de e para os recursos de catálogos de rquivos, bibliotecas e museus publicados na Web, é formada uma "nuvem", a "Linking Open Data Cloud". Vários autores consideram que a chave da semântica entre conteúdos de recursos informacionais reside em inter-relacioná-los (SHET et al., 2003).

A Ciência da Informação sempre se preocupou com a questão do controle semântico dos recursos informacionais, através das linguagens documentárias. Valoriza em especial as relações semânticas como fundamentais para agregar de semântica aos recursos informacionais e tem se dedicado a inventariá-las e estudá-las. Além disso, em ambientes digitais, relações são a essência da capacidade de computadores realizarem "inferências" automáticas. Na medida em que elementos de um artefato computacional estejam interrelacionados, como numa base de dados ou numa ontologia, um "agente de software" é capaz de realizar "inferências" automáticas, descobrindo conhecimento não diretamente explicitado, com base nessas relações, seguindo os "links" entre os nós de uma rede semântica (MARCONDES, 2011).

As tecnologias da Web Semântica e dados abertos interligados constituem o que muitos chamam de novo "paradigma" na interligação de acervos digitais: "The Europeana technical strategy thus was conceived to both contribute to and benefit from the growing Linked Data paradigm" (DOERR et al., 2010, p. 2). Esta caracterização será desenvolvida ao longo deste trabalho. Além disto, e talvez o que seja mais importante para instituições como arquivos, bibliotecas e museus, através delas é possível integrar conteúdos digitais de acervos de memória e cultura entre si e ao "mainstream" da web, formada de conteúdos e de "links" entre eles. Isto permitirá a estas instituições tirarem partido das sinergias e

\footnotetext{
${ }^{5}$ Disponível em: <http://lod-cloud.net>. Acesso em: 18 jun. 2016.
} 
complementaridades entre seus acervos e oferecerem melhores serviços prover informação cultural curada, tratada, contextualizada, de alta qualidade, aos seus usuários.

Outra possibilidade de integração de acervos heterogêneos criada a partir do surgimento destas tecnologias seria criar "links semânticos" relações - entre objetos digitais de acervos - representações digitais de objetos pertencentes a acervos em diferentes instituições arquivísticas, bibliotecas, museus, centros de documentação, cinematecas, coleções específicas. Um link semântico seria, por exemplo, ligar o registro do arquivo digital de um filme numa cinemateca digital com a imagem digital de uma pintura no acervo de um museu, que inspirou o filme. Em casos como o citado, estas relações têm relevância principalmente de um ponto de vista cultural.

No entanto, devido à longa tradição de trabalharem isoladamente e às especificidades e diversidades daí decorrentes, a questão dessa possível integração vai além da mera questão tecnológica. Na verdade, questões tecnológicas envolvendo a concepção de sistemas gerenciadores de acervos e de formatos de metadados "comuns"em arquivos, bibliotecas e museus têm sido apontadas como um problema para a integração destes acervos (OLDMAN et al., 2014). As experiências de interoperabilidade mais consistentes têm origem na área de bibliotecas, onde os acervos são bastante homogêneos. Quando se trata de integrar acervos de arquivos, bibliotecas e museus, ao contrário, a heterogeneidade dos acervos é bastante significativa.

Diante deste quadro de novas possibilidades trazidas pelas tecnologias da Web Semântica e dos dados abertos interligados este trabalho tem o objetivo de oferecer um quadro geral atualizado sobre a questão da interoperabilidade entre acervos digitais em arquivos, bibliotecas e museus, endereçando as seguintes questões. Qual a real complexidade para se atingir a interoperabilidade entre acervos heterogêneos de instituições que custodiam acervos digitais heterogêneos? Que papel jogam aí os modelos conceituais como Functional Requirements for Bibliographic Records (FRBR), Conceptual Reference Model/ International Committee for Documentation (CRM/CIDOC) ou Europeana Data Model (EDM)? Como modelos conceituais podem apoiar o uso destas tecnologias? Como instrumentalizar estes "links semânticos" com as tecnologias da Web semântica e dados abertos interligados, de modo a potencializar acervos tornados digitais em arquivos, bibliotecas e museus, de modo a potencializar suas sinergias? Que papel jogam as atividades de curadoria aplicadas à publicação de dados abertos interligados?

A metodologia utilizada foi a revisão do estado da arte com base na literatura a repeito da questão da interoperabilidade, incluindo aspectos teóricos, técnicos, projetos, experiências. Em Arms et al. (2002) é proposto um método para análise de soluções de interoperabilidade, baseado no grau de complexidade tecnológica e metodológica exigida de cada um dos sistemas a serem tornados interoperáveis versus as 
funcionalidades e benefícios advindo com a interoperabilidade. Este método no entanto não leva em consideração a heterogeneidade dos acervos a serem tornados interoperáveis, questão crítica no caso de integrar acervos em arquivos, bibliotecas e museus. Interoperabilidade é definido pela National Information Standard Organization (NISO) como "[...] the ability of multiple systems with different hardware and software platforms, data structures, and interfaces to exchange data with minimal loss of content and functionality." (NISO, 2004, p. 2). Quando se trata de sistemas absolutamente iguais, como no caso de começar a editar um texto no computador do trabalho usando o programa MSWord, leva-lo para casa salvo em um "pen-drive" e terminar sua edição à noite em meu "notebook" no mesmo programa MSWord, não existe problema de interoperabilidade. Estes só começam a existir na medida que queremos tornar interoperáveis sistemas diferentes. Portanto as soluções de interoperabilidade entre acervos como as endereçadas neste trabalho têm que preservar a semântica dos conteúdos originais dos acervos tornados interoperáveis. Tendo esta questão em vista foram utilizados dois parâmetros para a análise das diferentes soluções de interoperabilidade surgidas ao longo do tempo, a homogeneidade/heterogeneidade dos acervos a serem integrados e os mecanismos para garantir que os significados originais dos conteúdos nos sistemas isolados seriam preservados nos sistemas tornados interoperáveis.

O trabalho esta organizado como se segue. Após esta introdução a seção 2 revê a questão das relações semânticas na organização do conhecimento; a seção 3 discute os modelos de interoperabilidade baseados na troca de metadados. A seção 4 discute o potencial dos modelos conceituais nas soluções de interoperabilidade. A seção 5 discute as questões colocadas pela publicação de acervos como dados abertos interligados. Finalmente a seção 6 apresenta as considerações finais.

\section{Relações semânticas na organização do conhecimento}

Embora de forma esporádica, aparecem recorrentemente na literatura menções à questão das relações como expressões do significado e do conhecimento. Deixar claro o papel das relações é importante uma vez que as tecnologias de dados abertos interligados baseiam sua semântica em conteúdos interligados. Farradane (1980) ao propor sua Indexação Relacional, afirma: "Meaning, considered as relations between terms...". De acordo com Brookes (1980): "knowledge is a structure of concepts linked by their relations and information is a small part of such a structure". Sheth; Arpinar; Kashyap (2003) afirma que "Relationships are fundamental to semantics - to associate meaning to words, items and entities. They are a key to new insights. Knowledge discovery is about discovery of new relationships". Miller (1947) afirma que: "The above remarks imply that science is a search after internal relations between phenomena". 
Especificamente na área de Ciência da Informação, após o importante volume organizado por Bean e Green (2001), a própria American Society for Information Science and Technology (ASIST) lança um dos capítulos do ARIST de 2006 sobre o tema "Semantic Relations in Information Science" (KHOO; NA, 2006), indicando claramente a importância do tema para a área. Em 2006 Legg escreve uma revisão para a Annual Review of Information Science and Technology (ARIST) sobre a importância das ontologias para as questões de recuperação da informação e organização do conhecimento.

A organização do conhecimento discute exaustivamente a natureza das assim chamadas relações associativas, onde possivelmente estariam incluídas as relações que têm relevância principalmente de um ponto de vista cultural. Das relações associativas Peters e Weller (2008, p. 101), afirmam que "They are unspecified connections of concepts that can have any kind of relation", "Thesauri... make use of (entirely undifferentiated) associative relations", e ainda que "In addition, associative relations can be split into a diversity of domain-dependent, specified paradigmatic relations". Green (2008, p. 156) afirma que "Associative relations come into a variety of flavors".

As relações associativas são definidas por exclusão das relações hierárquicas ou paradigmáticas. Segundo o EuroVoc Thesauros, "The associative relationship is a relationship between two concepts which do not belong to the same hierarchical structure, although they have semantic or contextual similarities". Marcia Lei Zeng (2005) também as define de maneira similar: "This relationship covers associations between terms that are neither equivalent nor hierarchical, yet the terms are semantically or conceptually associated".

$\mathrm{Na}$ Organização do Conhecimento as relações associativas enquanto tais são semanticamente genéricas e dúbias. A literatura da área também as reconhece como relações altamente dependentes de contextos específicos. Para que possam ser úteis viabilizando inferências em ambientes computacionais devem ser desdobradas em relações com semântica clara, inequívoca e formal.

\section{Interoperabilidade baseada na troca de metadados}

Pode-se compreender interoperabilidade como a propriedade de sistemas diferentes (por ex. sistemas de gestão de bibliotecas digitais, instrumentos de pesquisa arquivísticos automatizados, sistemas de gestão de acervos museológicos), através de padrões tecnológicos, acordos ou propostas, de serem capazes de operar em conjunto, visando a execução de uma tarefa. As diferentes soluções de interoperabilidade utilizadas até hoje enfatizam o aspecto da troca ordenada de conteúdos de forma significativa; formatos de metadados como MARC e Dublin Core têm um papel fundamental nestas soluções de interoperabilidade.

Estas soluções de interoperabilidade podem ser classificadas com relação ao momento em que se dá esta troca de metadados. Metadados 
podem ser trocados antes de serem utilizados, isto é, podem ser primeiro, agregados em uma base de dados comum como pré-condição para seu uso; ou podem ser trocados simultaneamente ao momento de sua consulta e utilização. A primeira solução é a dos formatos MARC ou LILACS $^{6}$, utilizado para a montagem de um catálogo coletivo/base de dados comum, ou o uso do padrão de metadados Dublin Core para a coleta automática de metadados no protocolo OAI-PMH. A segunda solução é a utilizada pelo protocolo $Z 39.50$ ou, no mais recente protocolo SRU/SRW (MORGAM, 2004), que permitem, a partir de um programa cliente, a consulta e recuperação de registros MARC ou MODS ${ }^{7}$ ou Dublin Core, de vários sistemas de catálogos diferentes, como se fossem um único catálogo virtual.

Nestas soluções a interoperabilidade sintática é garantida por um específico formato de codificação de conteúdos, como a norma ISO2709 ${ }^{8}$ ou a linguagem $X M L^{9}$ na qual são codificados os metadados Dublin Core no protocolo OAI-PMH. Quanto à interoperabilidade semântica, no caso de formatos como o MARC, ela é garantida por padrões como o uso associado do formato com o CCAA2 - Código de Catalogação AngloAmericano, $2^{a}$. edição, que especificaria não só a semântica de cada campo como também as regras de como transcrevê-lo num registro bibliográfico. Este esquema vem sendo usado por bibliotecas do mundo inteiro há décadas. Deve ser lembrado que os sistemas a serem interoperados neste caso têm seus conteúdos completamente uniformes, formados por registros bibliográficos, descritos segundo o CCAA2.

No caso de do uso do padrão de metadados Dublin Core pode-se dizer que a interoperabilidade semântica é informal, já que o conteúdo de cada elemento é descrito informalmente; seria o caso de Autor no sistema 1 e Artista no sistema 2, ambos correspondendo ao elemento dc:creator. Isto acarreta problemas como no caso de se buscar a interoperabilidade entre acervos arquivísticos, bibliográficos e museológicos; ao contrário dos acervos bibliográficos tornados interoperáveis através de formatos como o MARC, este acervos são pouco uniformes. Aspectos como a granularidade - registros bibliográficos representam uma obra, que pode ter várias edições e exemplares, registros arquivísticos representam agrupamentos como fundos ou séries, registros museológicos representam objetos únicos -, são difíceis de serem contornados e unificados através de um padrão de metadados tão genérico como o Dublin Core. A adoção dos chamados Perfis de Aplicação - Aplication Profiles ${ }^{10}$, http://dublincore.org/documents/profile-guidelines/ - tem sido uma das formas de tratar o problema de aspectos específicos da descrição de

\footnotetext{
${ }^{6}$ Formato LILACS. Disponível em: <http://metodologia.lilacs.bvsalud.org/download/P/LILACS-2ManualDescricao-pt.pdf>. Acesso em : 18 jun. 2016.

${ }^{7}$ MODS. Disponível em: <http://www.loc.gov/standards/mods/>. Acesso em : Acesso em 18 jun. 2016.

8 INTERNATIONAL STANDARD ORGANIZATION. ISO2709 Information and documentation: format for information exchange. 2008. Disponível em: <http://www.iso.org/iso/catalogue detail.htm?csnumber=41319 $>$. Acesso em: 18 jun. 2016

${ }^{9}$ XML. Disponível em: <http://www.w3.org/XML/>. Acesso em: Acesso em 18 jun. 2016.

${ }^{10}$ Dublin Core Aplication profiels. Disponível em: http://dublincore.org/documents/profileguidelines/http://dublincore.org/documents/profile-guidelines/. Acesso em 18 jun. 2016.
} 
objetos de diferentes comunidades de usuários não contemplados pelo padrão Dublin Core. No entanto, o desdobramento do padrão Dublin Core em elementos específicos de um Perfil de Aplicação, como foi o caso dos objetos museológicos (PERKINS; SPINAZZE, 1999; MILLER, 1999) não resolve o problema específico da interoperabilidade entre acervos de arquivos, bibliotecas e museus - bastante heterogêneos entre si -, antes o agrava.

Recentemente novas arquiteturas com relação às bibliotecas digitais, o setor pioneiro na interoperabilidade, têm sido propostas. Estas novas propostas têm influência, naturalmente, na questão da interoperabilidade. A partir do Digital Library Manifesto, foi criado o DELOS Digital Library Reference Model (CANDELA et al., 2008). As novas tecnologias disponíveis, como a Web Semântica e a computação em grid ${ }^{11}$ juntamente como modelo de referência citado trouxeram novas arquitetura para as bibliotecas digitais, baseadas não mais em um sistema único, mas na modularização de componentes de software e na distribuição de recursos informacionais e computacionais. Estas novas propostas são conhecidas como arquiteturas ponto a ponto (P2P), arquiteturas orientadas a serviços (SoA), funcionando sobre uma infraestrutura tecnológica de computação em grid (AGOSTI; SCHEK; TÜRKER, 2004). Com relação a questão da interoperabilidade as novas arquiteturas não mais centralizam e agregam metadados e sim têm como base uma proposta descentralizada.

A arquitetura ponto a ponto funciona com diversas bibliotecas digitais que se organizam em uma rede para fornecerem seus serviços. A arquitetura se baseia na existência de índices comuns para os recursos disponíveis nas bibliotecas da rede. Quando uma consulta é feita a um dos nós da rede os índices são consultados e se este nó não dispõe do recurso, a consulta é repassada ao nó correspondente e o recurso recuperado e entregue ao usuário. A arquitetura ponto a ponto funciona como o serviço Napster de fornecimento de cópias de músicas. Ao invés das cópias estarem em um único repositório, estão distribuídas em vários, e o Napster simplesmente direciona a requisição por uma música ao repositório correspondente.

A arquitetura orientada a serviços tira partido de um padrão para oferta e integração de serviços da Web Semântica, o Web Service ${ }^{12}$. Um web service é um tipo de serviço oferecido através da Web que pode ser acionado por uma interface padronizada, acionada por regras descritas segundo o padrão Web Service Description Language (WSDL) ${ }^{13}$. Existe também um diretório de localização de serviços específicos, consultado através do protocolo. Os web services poderiam integrar serviços oferecidos através da Web como a localização de um livro em uma biblioteca, sua aquisição em uma livraria, a verificação do seu cartão de crédito no seu banco e a entrega do livro através de uma transportadora.

\footnotetext{
${ }^{11}$ Grid computing. Disponível em: <https://en.wikipedia.org/wiki/Grid computing>. Acesso em: 18 jun. 2016

${ }_{12}$ Web Services. Disponível em: <https://en.wikipedia.org/wiki/Web service>. Acesso em: 18 jun. 2016.

${ }^{13}$ WSDL. Disponível em: <http://www.w3.org/TR/wsdl>. Acesso em: 18 jun. 2016.
} 
Web services são complementados através de um serviço de diretório e localização de serviços disponíveis que funcionam segundo o padrão Wev Service, chamado Universal Description, Discovery and Integration $(\mathrm{UDDI})^{14}$

\section{Modelos conceituais e interoperabilidade}

As soluções de interoperabilidade vistas até agora, além de serem baseadas na troca de metadados, têm como característica o fato de que estes metadados são trocados na forma de registros. Registros são conjuntos de itens de informação que representariam instâncias de entidades. Se tomarmos um registro MARC podemos constatar que em um único registro podem estar representados mais do que uma única entidade. Em um registro típico recuperado do catálogo da LC do romance Dom Casmurro, de Machado de Assis (escritor brasileiro) podemos considerar como entidades distintas (no mínimo), a obra, sua específica edição em determinado ano, o autor, com suas datas de nascimento e morte, e o editor, com a cidade onde esta situada a empresa editora. Estas quatro entidades estão implícitas no registro bibliográfico. As regras do formato MARC e do CCAA2 não faziam distinção ou não tinham necessidade de distinguir ou de explicitar, as entidades presentes em um registro bibliográfico nem suas inter-relações. A representação em um registro, bibliográfico através de campos e seus conteúdos mascara e esconde as entidades e as relações existentes entre elas; nesta forma de representação "rasa" e redutora, relações são "comprimidas", transformadas em campos. Afinal, que entidade um registro MARC representaria? Estas limitações semânticas, entre outras, dos registros bibliográficos, já vêm sendo sentidas por bibliotecas tradicionais usuárias deste padrão (BE PART OF THE WEB, 2011).

Recentemente outro componente tem sido proposto como capaz de agregar semântica aos conteúdos trocados entre sistemas heterogêneos, como seriam os dados originários de acervos arquivísticos, bibliográficos e museológicos, os modelos conceituais como FRBR, CRM/CIDOC e EDM. Modelos conceituais são representações formais de um domínio em termos das classes de entidade e suas relações ai existentes. Quando se trata da interoperabilidade entre dois sistemas um modelo conceitual ajuda a estabelecer correspondências e diferenças ontológicas (GUARINO, 1997) entre as entidades representadas nos dois sistemas. No caso de modelos como o CRM/CIDOC, entidades locais aos dois sistemas são especializações das entidades do modelo conceitual. Seria o mesmo caso de Autor no sistema 1 e Artista no sistema 2; ambas as entidades seriam especializações da classe E39 Actor, do CRM/CIDOC. Para tornar isto possível entidades e relações devem estar explícitas.

A promessa dos modelos conceituais é facilitar a interoperabilidade entre acervos de diferentes instituições de memória e cultura. Em especial, este é um dos objetivos explícitos do Modelo Conceitual de

\footnotetext{
${ }^{14}$ UDDI. Disponível em: <http://pt.wikipedia.org/wiki/UDDI>. Acesso em: 18 jun. 2016
} 
Referência (CRM), do CIDOC/ICOM: "The CRM provides a core ontology that can harmonise between museum, archive, library, and other specialized cultural datasets" (OLDMAN et al., 2014, p. 1); e também: "The Intended Scope of the CRM may be defined as all information required for the exchange and integration of heterogeneous scientific documentation of museum collections...The exchange of relevant information with libraries and archives" (THE CIDOC CONCEPTUAL REFERENCE MODEL, 2013, p. ii).

A ênfase nas questões semânticas dos conteúdos em oposição a um específico formato de representação/disseminação destes conteúdos e a possibilidade de integrá-los a outros conteúdos em outros domínios também têm sido apontadas como uma vantagem dos modelos conceituais. É o que afirma Svensson (2013, p. 8):

A shift to a more model-driven view on bibliographic information would increase the possibilities to interlink the individual parts of a bibliographic record to other entities outside of the library domain, particularly within the cultural heritage sector, but also in settings like academia e ecommerce.

Um modelo conceitual genérico apoiaria assim um eventual mapeamento de dados de um sistema no outro ou a utilização conjunta de dados de ambos os sistemas, servindo de "ponte semântica" entre os sistemas 1 e 2; as entidades de ambos os sistemas seriam mapeadas nas entidades mais genéricas ou abstratas, do modelo conceitual. Para esta alternativa uma solução tecnológica é delineada em Kakali et al. (2007), na qual consultas a um sistema local são mapeadas para os elementos do modelo CRM/CIDOC e destes para os elementos do Dublin Core por um sistema mediador, transferidas para os outros sistemas locais que processariam as consultas e as respostas seriam consolidadas devolvidas para o usuário que fez a consulta.

Outro caso do uso de modelos conceituais é a biblioteca digital Europeana, http://www.europeana.eu. A Europeana funciona como um grande agregador de metadados coletados automaticamente a partir de diversos provedores de dados (TZOUVARAS et al., 2012) através do protocolo OAIPMH. Estes metadados são processados e enriquecidos com novos metadados com os recursos disponíveis na Europeana, como tesauros e arquivos de autoridades, gerando um formato interno capaz de representar objetos digitais complexos (isto é, formados por diversos arquivos digitais interligados) baseado no padrão Object Exchange and Reuse (ORE), https://www.openarchives.org/ore/, que, por sua vez, é baseado na tecnologia de dados abertos interligados, RDF. A base de todo este processamento interno e reformatação dos metadados coletados pela Europeana é um modelo conceitual, o Europeana Data Model (EDM) (DEFINITION OF THE EUROPEANA DATA MODEL, 2015). O EDM fornece a semântica das classes e relações (CONCORDIA; GRADMANN; SIEBINGA, 
2010) que formam os objetos digitais complexos codificados segundo as tecnologias de dados abertos interligados, especificamente os sujeitos, predicados e objetos, codificados como triplas RDF. Além de prover acesso direto a aos conteúdos nela armazenados a Europeana ainda dissemina a terceiros seus conteúdos através de API (interfaces de programas aplicativos) específicas, permitindo amplo reuso de seus dados.

Modelos conceituais, por terem semânticas específicas para suas entidades e a maneira como estas se interrelacionam, também são importantes em soluções de interoperabilidade baseadas nas tecnologias de dados abertos interligados. Eles fornecem um vocabulário com semântica formal para estas entidades e seus relacionamentos. O diretório Linked Open Vocabularies, http://lov.okfn.org/, traz vários vocabulários especificando entidades e relacionamentos baseados na semântica fornecida por modelos conceituais como FRBR e CIDOC CRM; este vocabulários são especificados em RDF e com URI específicas, ou seja, estão prontos para serem utilizados com as tecnologias de dados abertos interligados.

\section{Publicação de acervos como dados abertos interligados: novas questões}

As alternativas convencionais de interoperabilidade são baseadas, de uma forma ou de outra, na troca de metadados e no pressuposto de que exista uma equivalência semântica, parcial ou total, entre as (representações de) entidades de dois sistemas. No entanto uma eventual equivalência semântica não é o único caso de possíveis relações entre objetos de sistemas distintos. Existe todo um conjunto de outras relações como, por os exemplos que se seguem.

- A tela "Iracema", de José Maria de Medeiros, datada de 1881, que faz parte do acervo do Museu Nacional de Belas Artes do Rio de Janeiro, foi inspirada no romance homônimo, de José de Alencar, publicado pela primeira vez em 1865, que possui vários exemplares no acervo da Biblioteca Nacional.

- A Academia Brasileira de Letras possui em seu acervo museológico a escrivaninha onde Machado de Assis trabalhava e escreveu algumas de suas obras mais importantes, facilmente encontráveis em diversas bibliotecas brasileiras.

- O romance Dona Flor e seus dois maridos, de Jorge Amado, é ilustrado com gravuras do artista Carybé, cujos originais se encontram na Coleção da Fundação Jorge Amando, Salvador, BA

- O livro "The Work of Oscar Niemeyer" de Stamo Papadaki publicado em 1950 em Nova York pela Editora "Reinhold Publishing" faz parte do acervo da Biblioteca da Faculdade de Arquitetura e Urbanismo da UFRJ, dentre outras no Brasil. Ele apresenta diversos projetos do arquiteto Oscar Niemeyer, dentre os quais, uma de suas primeiras residências concebidas, a residência de fim de semana para Oswald de Andrade. A perspectiva em nanquim sob papel vegetal, desenhada por Niemeyer, 
ilustra este livro, fazendo parte do Fundo Jorge Machado Moreira, sob custódia do Núcleo de Pesquisa e Documentação da Faculdade de Arquitetura e Urbanismo da UFRJ.

- A Peça de Indumentária utilizada pela cantora brasileira Carmen Miranda no filme "That Night in Rio", 1941, faz parte do Museu Carmen Miranda; cópia do filme pode ser encontrada na Coleção Rui Castro.

Estes são alguns casos mais significativos, levantados a partir de depoimentos de curadores de acervos em arquivos, bibliotecas e museus, relatando relações culturamente significativas entre objetos arquivísticos, bibliográficos e museológicos pertencentes a acervos de diferentes instituições. Estes casos foram levantados em um projeto exploratório de iniciação científica, orientado por nós (MARCONDES, 2012).

Inúmeros casos significativos são conhecidos, como o ballet Romeo e Julieta, do compositor russo Prokofiev, a obra musical abertura Hamlet, de Tchaikovsky, a versões da Mona Lisa, de artistas como o francês Marcel Duchamp, o americano Andy Warhol, Salvador Dali e o colombiano Botero. Estes, ao contrário da relação Obra-Expressão, prevista no Modelo FRBR, seriam talvez relações de tipo Obra inspirada em Obra. Outros exemplos seriam a Guernica de Picasso e os inúmeros esboços da obra feitos pelo artista, as obras de Van Gogh, as cartas do artista ao seu irmão Theo e a música Starry Night de Don McLean, obras arquitetônicas e obras de arte que as decoram, como os murais Guerra e Paz do artista brasileiro Cândido Portinari, que decoram o prédio sede da ONU em Nova Iorque, versões em cinema de famosas obras literárias, o ballet Parade, com música de Erik Satie, cenários de Jean Cocteou e figurinos de Picasso, famosos artistas plásticos que foram ilustradores de obras literárias. Os exemplos são inumeráveis na história das artes, etc, etc, etc.

Em artigo relatando a experiência de compatibilização dos modelos conceituais FRBR e CRM/CIDOC desenvolvida pela IFLA e pelo CIDOC/ICOM de 2003 a 2008, os autores chamam a atenção para algumas destas novas relações: "The work on this model revealed an important generic form of creative activity, the "incorporation" of an expression of a work in the expression of a work of another form or kind" (DOERR; BEKIARI; LEBOEUF, 2008).

Também têm que ser investigadas as possíveis relações indiretas entre objetos digitais nos acervos e recursos como os verbetes específicos da Wikipedia, https://www.wikipedia.org/, sobre personalidades, eventos históricos, lugares, etc., registros de arquivos de autoridades, como o Viaf, http://viaf.org/, ou recursos como Geonames, http://www.geonames.org/ ou 0 Getty Thesaurus of Geographic Names, http://www.getty.edu/research/tools/vocabularies/tgn/. Considerando estas relações indiretas, o espectro de relações culturalmente significativas se amplia bastante.

Os exemplos citados acima indicam que eventualmente possam existir relações culturalmente significativas entre objetos de acervos de arquivos, bibliotecas e museus. Nestes casos, objetos digitais representando objetos de acervos diferentes ou os itens de informação 
que os descrevem não tem equivalência semântica, mas demonstram terem relações válidas sob um ponto de vista cultural.

Relações como as citadas potencializam-se na medida que objetos destes acervos passam a ser representados como objetos digitais e disponibilizados na Web. A Web traz o potencial de integrá-los todos e integrá-los ainda com lugares, pessoas, eventos, épocas, estilos, movimentos artísticos, culturais, políticos e sociais.

Além disso, e talvez o que seja mais importante para instituições como arquivos, bibliotecas e museus, a disponibilização dos objetos digitais de seus acervos na Web através de tecnologias de dados abertos interligados viabiliza a exploração de relações com os mais diversificados conteúdos, seja de agências de turismo, eventos, músicas, vídeos, notícias, cursos ou disciplinas acadêmicas, mídias sociais, etc.

Falar em relações culturamente significativas entre objetos arquivísticos, bibliográficos e museológicos pertencentes a diferentes acervos significa reconhecer o valor e potencial cultural destas relações. É um trabalho de curadoria, naturalmente dentro de um novo contexto. "A curator (from Latin: curare meaning "take care") is a manager or overseer. Traditionally, a curator or keeper of acultural heritage institution (i.e., gallery, museum, library or archive) is a content specialist responsible for an institution'scollections and involved with the interpretation of heritage material. The object of a traditional curator's concern necessarily involves tangible objects of some sort, whether it is artwork, collectibles, historic items or scientific collections" ${ }^{15}$. Curadoria pode ser definida como a atividade de interpretação e agregação de valor de diferentes elementos que compõe, de alguma maneira, a herança cultural ou acervos específicos. Mais recentemente vem sendo cada vez mais empregado o termo "curadoria digital" embora com um uso um tanto quanto metafórico em relação ao significado tradicional, envolvendo a preservação dos dados de pesquisa digitais: "Digital curation involves maintaining, preserving and adding value to digital research data throughtout its life cycle"16. O problema colocado por estas relações culturalmente significativas retoma 0 sentido tradicional do termo curadoria, agora lançando mão, não de acervos físicos, mas sim, de suas representações digitais publicadas na Web.

Como caracterizar com mais clareza o que aqui são chamadas de relações culturalmente significativas entre objetos de acervos de arquivos, bibliotecas e museus? Até que ponto modelos conceituais como os utilizados na área de documentação e informação endereçam estas relações?

O modelo conceitual FRBR prevê relações entre obras, suas manifestações, suas expressões e os itens específicos correspondentes às mesmas, armazenados num acervo bibliográfico; por exemplo, o Hamlet de Shakespeare, suas diversas manifestações enquanto textos das peças

\footnotetext{
${ }^{15}$ Curator. In: WIKIPEDIA the Free Encyclopedia. [s.d]. Disponível em: < http://en.wikipedia.org/wiki/Curator>. Acesso em: 18 jun. 2016

${ }^{16}$ Digital Curation Center. Disponível em: <http://www.dcc.ac.uk/>. Acesso em: 18 jun. 2016.
} 
ou filmes, as específicas expressões destas manifestações, como o filme em que o ator Mel Gibson interpreta Hamlet ou o texto da peça editado pela Penguin Books, e os itens específicos dessas expressões. Estas relações endereçam algumas das que citamos. No entanto no modelo FRBR obras e manifestações são entidades abstratas, enquanto falamos de representações digitais de objetos nos diferentes acervos, que seriam relações manifestação-manifestação.

O padrão de metadados Dublin Core (http://dublincore.org), embora não possa ser considerado com modelo conceitual, possui entre seus elementos "Source", definido como "A Reference to a resource from which the present resource is derived". Usando o elemento dc:source, várias manifestações de uma obra poderiam ser relacionadas.

O modelo CRM contempla entre seus entre seus objetivos a integração e interoperabilidade entre acervos de arquivos, bibliotecas e museus: "The CIDOC Conceptual Reference Model ("CRM"), a formal ontology intended to facilitate the integration, mediation and interchange of heterogeneous cultural heritage information" (THE CIDOC CONCEPTUAL REFERENCE MODEL, 2013, p. i). E ainda "The CRM should become our language for semantic interoperability" (DOERR; STEAD, 2008, p. 62).

O CRM reconhece a importância das relações como portadoras de significado: "Understanding lives from relationships" (DOERR; STEAD, 2008, p. 3).

Um exame inicial, não exaustivo, indica que as seguintes relações no CRM CIDOC poderiam endereçar algumas das relações culturalmente significativas previamente mencionadas.

a)P148, has component (is component of), entre a expressão de uma obra e suas partes;

b)P70, documents (is documented in), entre um documento e objeto documentado por este;

c) P62, depicts (is depicted by), entre um artefato e o objeto por ele descrito;

d)P15, was influenced by (influenced), entre uma atividade e um objeto influenciado pela mesma, e uma de suas subpropriedades, P17, was motivated by (motivated).

Também têm que ser investigadas as possíveis relações indiretas entre objetos digitais nos acervos e recursos como os verbetes específicos da Wikipedia, sobre personalidades, eventos históricos, lugares, etc., registros de arquivos de autoridades, como o Viaf, ou recursos como Geonames. Considerando estas relações indiretas, o espectro de relações culturalmente significativas se amplia bastante.

Outra questão importante. O artigo de Pattuelli et al. (2013) relata a experiência do Projeto Linked Jazz, http://linkedjazz.org/ da publicação de seus dados abertos interligados. Este relato deixa claro que não basta às 
instituições de memória e cultura publicarem seus acervos como dados abertos interligados. Todo um esforço adicional deve ser feito no sentido de viabilizar a criação de novos "links", de outras relações culturamente significativas, com outros recursos também disponíveis na Web, além daquelas tradicionais e já representadas nos catálogos convencionais de arquivos, bibliotecas e museus, como autor e sua obra, uma obra e seus colaboradores (tradutores, editores, etc).

Frequentemente o que tem sido feito é ligar os dados dos catálogos com os verbetes da Wikipedia, os registros de autoridades como o Viaf ou com o Geonames, como mencionado anteriormente. Embora a Wikipedia tenha um papel fundamental neste caso, nem ela nem o Viaf trazem uma compilação de todos os títulos ou, pelo menos, da maior parte, de obras das artes plásticas, ou da literatura, das artes cênicas ou da música, de um determinado criador. Tal complemento da Wikipedia, do Viaf, ou a criação de outro recurso semelhante, relacionando criador e o título de suas obras, seria muito útil para facilitar a interligação de dados abertos como os vários exemplos citados no início desta seção e que foram chamadas de relações culturalmente significativas. Instituições que tem um mandato de curadores deste legado cultural, como bibliotecas nacionais, museus de artes, etc. deviam tomar a si este papel e complementarem como sugerido recursos como a Wikipedia e o Viaf.

\section{Considerações finais}

As soluções de interoperabilidade evoluíram da seguinte maneira ao longo do tempo, em relação às questões que nos interessam, a heterogeneidade de acervos e a preservação da semântica dos conteúdos tornados interoperáveis:

- Soluções de interoperabilidade baseadas na troca e agregação de metadados:

- Troca de dados baseada em registros de formatos padronizados, como MARC e LILACS, para tratamento de acervos altamente homogêneos, com uma semântica implícita, baseada no CCAA2.

- Troca de dados baseada em registros de formatos padronizados, como o Dublin Core, para tratamento de acervos tornados homogêneos através dos 15 elementos DC, mas também vista como solução para acervos heterogêneos, tratados através da qualificação dos elementos DC ou da criação de perfis de aplicação específicos, http://dublincore.org/documents/profile-guidelines/; a semântica é implícita quando se trata somente dos 15 elementos DC ou, mais explícita quando usados os elementos DC qualificados ou os perfis de aplicação.

- Modelos conceituais como enriquecedores semânticos, orientando a conversão de metadados convencionais em representações mais ricas semanticamente.

- Modelos conceituais como mediadores semânticos entre recursos independentes, permitindo a consulta a um conjunto de recursos heterogêneos a partir de uma visão única dada pelo modelo conceitual. 
- Soluções de interoperabilidade descentralizadas, não baseadas na troca, agregação e padronização de metadados:

- Arquiteturas ponto a ponto ou orientadas a serviços.

- Publicação de conteúdos diretamente na Web e na sua interligação através de "links semânticos"; a semântica dos conteúdos e dos links é dada por modelos conceituais e/ou vocabulários específicos; tecnologia de dados abertos interligados.

Para que possam tirar partido de todas as possibilidades abertas com a publicação de conteúdos diretamente na Web, os modelos conceituais que orientam e dão semântica ao processo de publicação na Web devem contemplar as visões dos possíveis usuários que atualmente são minimizadas em função de um jargão profissional. Discutindo a importância das bases de dados de autoridades nas possíveis interligações entre acervos, Svensson (2013, p. 10) afirma que: "In LoC-NAF John Lewis Burckhardt is both a name (madsrdf:PersonalName) and a skos:Concept. This is in line with cataloging tradition, but a non-librarian would be surprised that rdf:type is not, for example, foaf:person".

Também é importante para o objetivo de se atingir a interoperabilidade entre acervos de instituições de memória e cultura o trabalho que vem sendo desenvolvido pelo CIDOC/ICOM, pela IFLA e pelo ICA, de desenvolvimento de novos modelos conceituais (GUEGUEN et al., 2013) e compatibilização entre estes (DOERR; LEBOEUF, 2007).

A Web e os formatos digitais de conteúdos tornam as instituições de memória e de cultura seus acervos acessíveis a um público muito mais amplo do que seria possível de forma presencial. A Rede vem se tornando um instrumento sem precedentes para a disseminação desses acervos, a um público mais amplo, a qualquer hora, de qualquer lugar, através de dispositivos como computadores convencionais até "smartphones" (HSI, 2002). A Web pode contribuir assim para democratizar o acesso a estes conteúdos, antes só disponíveis ao público presencial. A possibilidade de disseminarem seus acervos através da Web amplia os papeis e justificativas sociais, educacionais e culturais das instituições de memória. Hoje se torna cada vez mais necessário que estas levem em conta a Web e suas possibilidades em suas políticas.

A Web oferece assim um novo espaço para a curadoria desses acervos digitais culturalmente relacionados, tirando partido de suas sinergias e ampliando suas potencialidades culturais e educativas. As soluções tecnológicas devem vir acompanhadas de soluções de curadoria. Estas potencialidades para a cultura e educação são inimagináveis, como nunca antes, em nenhum período da história, existiram. A este respeito Clough (2013, p. 5) afirma: "And by facilitating partnerships and collaborations among institutions, digital technology offers the public a streamlined way to access information and take advantage of powerful, jointly curated exhibitions".

As questões levantadas mostram que a interoperabilidade entre acervos de arquivos, bibliotecas e museus vai além de uma solução meramente tecnológica. Questões específicas de interesse das instituições 
de memória e cultura devem ser endereçadas, algumas delas apontadas neste trabalho, e pesquisas aprofundadas à respeito. A publicação de acervos de arquivos, bibliotecas e museus diretamente na Web usando as tecnologias da Web Semântica e de dados abertos interligados permite a estas instituições tirarem partido das sinergias múltiplas. Estas soluções são descentralizadas, tirando partido de um formato unificador mas não redutor como RDF e vão assim na direção contrária das soluções de interoperabilidade baseadas na agregação, centralização e padronização de metadados. Tornam portanto maiores as demandas pela preservação da semântica dos conteúdos tornados interoperáveis e aí, passam a ter papel fundamental os modelos conceituais e as novas práticas e recursos de curadoria em ambiente Web.

\section{Referências}

AGOSTI, M.; SCHEK, H. J.; TÜRKER, C. Digital library architectures: peerto-peer, grid, and service-orientation. In: THEMATIC WORKSHOP OF THE EU NETWORK OF EXCELLENCE DELOS, 6., S. Margherita Di Pula, Cagliari, Italy, 24-25 June, 2004. Pre-proceedings... Italy, 2004. Disponível em: <http://www.researchgate.net/profile/Leonardo_Candela/publication/200 462074_Moving_Digital_Library_Service_Systems_to_the_Grid/links/Ofcfd 50519eff1bed6000000.pdf>. Acesso em: 18 jun. 2016.

ARMS, W. Y. et al. A Spectrum of interoperability: the site for science for prototype for the NSDL. D-Lib Magazine, v. 8, n. 1, 2002. Disponível em: <http://www.dlib.org/dlib/january02/arms/01arms.html>. Acesso em: 18 jun. 2016.

BE PART OF THE WEB. Report of the Stanford Linked Data Workshop, 27 June. 1 July 2011. Disponível em: <http://www.clir.org/pubs/abstract/pub152abst.html>. Acesso em: 18 jun. 2016.

BEAN, C. A.; GREEN, R. (Eds.). Relationships in the Organization of Knowledge. Dordrecht: Kluwer Academic Press, 2001. (Information Science and Knowledge Management, v. 2).

GREEN, R. Relationships in the Organization of Knowledge. Knowledge Organization, v. 35, n. 2/3, p. 150-159, 2008.

BROOKS, B. The foundations of information science. Part I. Philosophical aspects. Journal of Information Science, v. 2, p. 125-133, 1980.

CANDELA, L. et al. The DELOS Digital Library Reference model: foundations for digital libraries (Version 0.98). 2008. Disponível em: <http://eprints.port.ac.uk/4104/>. Acesso em: 12 jun. 2015.

CLOUGH, W. Best of both worlds: museums, libraries and archives in a digital age. Smithsonian Institute, 2013. Disponível em: <http://www.si.edu/content/gwc/bestofbothworldssmithsonian.pdf>.

Acesso em: 2 fev. 2015. 
CONCORDIA, C.; GRADMANN, S.; SIEBINGA, S. Not just another portal, not just another digital library: A portrait of Europeana as an application program interface. IFLA journal, v. 36, n. 1, p. 61-69, 2010.

DEFINITION OF THE EUROPEANA DATA MODEL. v5.2.5. 2015. Disponível em: <http://pro.europeana.eu/documents/900548/0d0f6ec3-1905-4c4f96c8-1d817c03123c>. Acesso em: 15 nov. 2014.

DOERR, M. et al. The europeana data model (EDM). In: WORLD LIBRARY AND INFORMATION CONGRESS: IFLA GENERAL CONFERENCE AND ASSEMBLY, 76., 2010. Proceedings... 2010. p. 10-15. Disponível em: <http://kennison.name/files/zopestore/uploads/metadata/europeana/pres entation.pdf $>$. Acesso em: 13 set. 2014.

DOERR, M.; LEBOEUF, P. Modelling intellectual processes: the FRBR-CRM harmonization. In: DIGITAL libraries: research and development. Berlin Heidelberg: Springer, 2007. p. 114-123. Disponível em: <http://network.icom.museum/fileadmin/user_upload/minisites/cidoc/Con ferencePapers/2006/Doerr_LeBoeuf.pdf>. Acesso em: 13 set. 2014.

DOERR, M.; BEKIARI, C. ; LEBOEUF, P. Conceptual model for performing arts. In: ICOM INTERNATIONAL COMMITTEE FOR DOCUMENTATION, September 2008, Athens. Proceedings... Athens, 2008. Disponível em: <http://www.cidoc-crm.org/docs/2008-09-12_PerformingArts.pdf>.

Acesso em: 13 set. 2014.

FARRADANE, J. Relational indexing. Part I. Journal of Information Science, v. 1, p. 267-276, 1980.

GUARINO, N. Some organizing principles for a unified top-level ontology. In: AAAI Spring Symposium on Ontological Engineering. 1997. p. 57-63. Disponível em: <http://www.aaai.org/Papers/Symposia/Spring/1997/SS97-06/SS97-06-008.pdf>. Acesso em: 3 mar. 2008.

GUEGUEN, G. et al. Para um modelo conceitual internacional de descrição arquivística. Acervo, v. 26, n. 2, 2013. Disponível em: $<$ http://www.revistaacervo.an.gov.br/seer/index.php/info/article/view/658>. Acesso em: 27 maio 2015.

HEATH, T.; BIZER, C. Linked data: evolving the web into a global data space. Synthesis lectures on the semantic web: theory and technology, v. 1, n. 1, p.1-136, 2011. Diposnível em: <http://www.morganclaypool.com/doi/abs/10.2200/s00334ed1v01y2011 02wbe001>. Acesso em: 2 jun. 2012.

HSI, S. The electronic guidebook: a study of user experiences using mobile web content in a museum setting. In: IEEE INTERNATIONAL WORKSHOP. Wireless and Mobile Technologies in Education, 2002. Proceedings... IEEE, 2002. p. 48-54.

KAKALI, C. et al. Integrating Dublin Core metadata for cultural heritage collections using ontologies. In: INTERNATIONAL CONFERENCE ON DUBLIN CORE AND METADATA APPLICATIONS, Singapure, 2007. 
Proceedings... Singapure: DCMI, 2007. p. 128-139. Disponível em: $<$ http://dcpapers.dublincore.org/index.php/pubs/article/viewFile/871/867 $>$. Acesso em: 24 maio 2015.

KHOO, C. S. G.; NA, J.-C. Semantic relations in information science. Annual Review of Information Science and Technology, v.40, p. 157-228, 2006.

LEGG, C. Ontologies on the Semantic Web. Annual Review of Information Science and Technology, v. 40, p. 157, 2006.

MARCONDES, C. H. O papel das relações semânticas na organização do conhecimento em ambientes digitais. In: SILVA, F. C. C.; SALES, R. (Orgs). Cenários da organização do conhecimento: linguagens documentárias em cena. Brasília: Thesaurus, 2011. p. 129-168.

MARCONDES, C. H. "Linked data" e interoperabilidade entre arquivos, bibliotecas e museus na web. Niterói: PROPi/UFF, 2012. (Projeto de Iniciação Científica submetido e aprovado pelo Programa Institucional de Bolsas de Iniciação Científica PIBIC - UFF 2012).

MILLER, D. L. Explanation versus description. Philosophical Review, v. 56, n. 3, p. 306-312, 1947.

MILLER, P. Interoperability: what is it and why should I want it. Ariadne, v. 24, 2000. Disponível em: <http://www.ariadne.ac.uk/issue24/>. Acesso em: 25 jan. 2001.

MILLER, P. Dublin Core for Museum. In: CONSORTION for The Computer Interchange of Museum Information, Interoperability focus (CIMI). Taiwan: UKOLN, 1999. [slides]. Disponível em: <http:// www.ukoln.ac.uk/interop-focus/presentations/.../taiwan/.../day1.ppt>. Acesso em: 18 jun. 2016.

MORGANB, E. L. An introduction to the search/retrieve URL service (SRU). Ariadne, $\quad$ v. 40, 2004. Disponível em: <http://www.ariadne.ac.uk/issue40/morgan/>. Acesso em: 15 jan. 2009.

NILSSON, M.; BAKER, T.; JOHNSTON, P. Interoperability levels for Dublin Core Metadata. Dublin Core Metadata Initiative. (DCMI recommended resource). $2009 . \quad$ Disponível em: <http://dublincore.org/documents/2009/05/01/interoperability-levels/>.

Acesso em: 13 maio 2015.

NISO. Understanding metadata. Bethesda (EUA): National Information Standards Organization, 2004. Disponível em: <http://www.niso.org/publications/press/UnderstandingMetadata.pdf>. Acesso em: 13 mai. 2015.

OLDOMAN, D. et al. Realizing lessons of the last 20 years: a manifesto for data provisioning \& aggregation services for the digital humanities (a position paper). D-lib magazine, v. 20, n. 7, p. 6, 2014. Disponível em: <http://www.dlib.org/dlib/july14/oldman/07oldman.html>. Acesso em: 27 jun. 2015. 
PATTUELLI, M. C. et al. Crafting linked open data for cultural heritage: mapping and curation tools for the linked jazz project. Code4Lib Journal, v. 21, 2013. Disponível em: <http://journal.code4lib.org/articles/8670>. Acesso em: 2 mar. 2016.

PERKINS, J.; SPINAZZE, A. T. Finding museum information in the internet commons: a report on the CIMI Dublin Core Metadata Testbed Project. In: INTERNATIONAL CULTURAL HERITAGE INFORMATICS MEETING, 5., Washington DC, 1999. Proceedings... Washington, 1999. p. 175-177.

PETERS, I.; WELLERS, K. Paradigmatic and syntagmatic relations in knowledge organization. Information Wissenschaft \& Praxis, v. 59, n. 1, p. 100-107, 2008.

RIBEIRO, F. Arquivística como disciplina aplicada no campo da Ciência da Informação. Perspectivas em Gestão \& Conhecimento, João Pessoa, v. 1, n. 1 , p. 59-73, 2011. Disponível em: <http://periodicos.ufpb.br/ojs2/index.php/pgc/issue/current/showToc>. Acesso em: 3 mar. 2015.

RESORCE DESCRIPTION FRAMEWORK (RDF). W3C Semantic Web. 2014. Disponível em: < https://www.w3.org/RDF/ >. Acesso em 18 jun. 2016.?

ROBREDO, J.; CUNHA, M. B. Documentação de hoje e de amanhã: uma abordagem informatizada da Biblioteconomia e dos sistemas de informação. São Paulo: Global, 1986.

RODRÍGUEZ, E. M. M.; GREENBERG, J. Linked data for open vocabularies and HIVE's global framework. El profesional de la información, v. 21, n. 3, p. 236-244, 2012. Disponível em: <http://dx.doi.org/10.3145/epi.2012.may.03>. Acesso em: 13 jan. 2013.

SVENSSON, L. G. (2013). Are current bibliographic models suitable for integration with the web. Information Standards Quarterly, Winter, v. 25, n. 4, p. 6-13, 2013. Disponível em: <http://www.niso.org/apps/group_public/download.php/11931/FE_Svens son_bibliographic_models_isqv25no4.pdf>. Acesso em 13 jun. 2015.

SHETH, A.; ARPINAR, I. B.; KASHYAP, V. Relationships at the heart of semantic web: modeling, discovering and exploiting complex semantic relationships. In: M. NIKRAVESH, M. et al. (Eds.). Enhancing the power of the internet: studies in fuzziness and soft computing. Springer-Verlag, 2003. Disponível em: <http//cgsb2.nlm.nih.gov/ kashyap/publications/relations.pdf>. Acesso em: 24 maio 2007.

THE CIDOC CONCEPTUAL REFERENCE MODEL. Version 5.1.2. ICOM/CIDOC CRM Special Interest Group, 2013. Disponível em:

TZOUVARAS, V. et al. Report on semantic interoperability with europeana: ECP 2008. DILI 518002. EUscreen: eContentplus Programme, 2012. Disponível em: <http://pro.europeana.eu/files/Europeana_Professional/Projects/Project_li 
st/EUscreen/Deliverables/EUscreen\%20D4.8_ReportOnSemanticInteroper abilityWithEuropeana.pdf>. Acesso em: 31 maio 2015.

WALCH, V. I. Standards for archival description: a handbook. Society of Americam Archivists, 1994. Disponível em: <http://www.archivists.org/catalog/stds99/index.html>. Acesso em: 2 abr. 2015. 